\title{
Autologous Fat Grafting in the Treatment of Fibrotic Perioral Changes in Patients With Systemic Sclerosis
}

\author{
Nicoletta Del Papa, ${ }^{*}$ Fabio Caviggioli, $†$ Domenico Sambataro, ${ }^{*}$ Eleonora Zaccara, ${ }^{*}$ Valeriano Vinci, \\ Gabriele Di Luca,§ Antonina Parafioriti,II Elisabetta Armiraglio,I[ Wanda Maglione,* Riccardo Polosa,\# \\ Francesco Klinger, $\dagger$ and Marco Klinger $\ddagger$ \\ *U.O.C. Day Hospital Reumatologia, Ospedale G. Pini, Milano, Italy \\ †U.O.C. Chirurgia Plastica, Multimedica Holding SpA, Milano, Italy \\ ¥U.O.C. Chirurgia Plastica, Istituto Clinico Humanitas, Università degli studi di Milano, Milano, Italy \\ §U.O.S. Chirurgia Vascolare, Ospedale G. Pini, Milano, Italy \\ IUU.O.C. Anatomia Patologica, Ospedale G. Pini, Milano, Italy \\ \#Istituto di Medicina Interna e d'Emergenza, Dip. Biomedicina Clinica e Molecolare, Università degli Studi di Catania, Catania, Italy
}

\begin{abstract}
Autologous fat tissue grafting (AFTG) has been successfully used in the treatment of different sclerotic conditions, including localized scleroderma. Patients with advanced systemic sclerosis (SSc)-related perioral thickening and mouth opening limitation are candidates for this therapeutic approach. AFTG of the lips was performed to improve mouth opening in patients with SSc. We enrolled in the study 20 female patients with diffuse SSc (median age $35 \pm 15$ years and $11 \pm 10$ years of disease duration). Two-milliliter fractions of autologous fat drawn from trochanteric or periumbilical areas were injected in eight different sites around the mouth. Baseline and after-treatment mouth opening changes were assessed by measuring interincisal distance and oral perimeter, while skin hardness was tested by digital durometer. Pre- and posttreatment modifications of microvascular architecture were assessed by counting capillaries in the inferior lip videocapillaroscopy (VC) images and by scoring the microvascular density (MVD) in anti-CD34/CD31 immunohistochemical (IH) stained perioral skin biopsy sections. Similarly, histological sections were examined to evaluate dermoepidermic junction (DEJ) modifications. Three months after treatment, both the interincisal distance and oral perimeter significantly increased $(p<0.001)$. At the same time, a significant skin neovascularization became evident, both considering the VC images $(p<0.001)$ and MVD scores in IH sections $(p<0.0001)$. Finally, some skin histological aspects also improved, as shown by the significant changes in DEJ flattening scores $(p<0.0001)$. The present study suggests that, in patients with SSc, AFTG can improve mouth opening and function, induce a neovascularization, and partially restore the skin structure.
\end{abstract}

Key words: Systemic sclerosis (SSc); Adipose tissue; Stem cells; Autologous graft

\section{INTRODUCTION}

Systemic sclerosis (SSc) is classically included among the autoimmune systemic disorders. Immune system dysregulation, with T-/B-cell imbalance and production of autoantibodies, and endothelial cell activation, with the consequent microvascular damage, certainly play an important role in the pathogenesis of this disorder. However, the hallmark of the disease is mainly represented by the activation of fibroblast lineage with overproduction of extracellular matrix and collagen in the skin and internal organs and by the related progressive fibrotic changes in the involved tissues (4). In both of the two distinct variants of this disorder, that is, diffuse and limited SSc, the involvement of the face skin is common (20). The loss of elasticity and the thickening of the skin in the perioral area and lips, besides the aesthetic damage, may cause a certain degree of disability since eating solid food, drinking, and personal or dentist care of the teeth may become difficult (15). Besides the other cutaneous, vascular, and systemic manifestations of SSc, this can certainly contribute to further compromise the quality of life of the patients (22).

In recent decades, autologous fat tissue grafting (AFTG) has been successfully used to treat a progressively larger number of clinical conditions characterized by skin atrophy or fibrosis, such as radiodermitis, posttraumatic or burning sequelae, breast reconstruction after surgery, and

Received June 25, 2013; final acceptance September 30, 2013. Online prepub date: October 22, 2013.

Address correspondence to Nicoletta Del Papa, M.D., Day Hospital Reumatologia, Ospedale G. Pini, Via Pini, 9 - 20122 Milano, Italy.

Tel: +39-0258296663; Fax: +39-0258296495; E-mail: delpapa@gpini.it 
craniofacial deformities $(6,8,19,32,34)$. More recently, some patients affected by linear scleroderma and different types of morphea have been treated by means of AFTG or by transplantation of adipose-derived mesenchymal stem cells (ATDMSCs) $(10,17,18,33,35)$. In most of these cases, a significant increase of skin elasticity and thickening has been reported, with both aesthetic and functional improvement of the treated skin areas, while no serious side effects have been observed during and after these kinds of procedures. The advantage of using adipose tissue or its derivates is the biocompatibility, with lower morbidity in comparison with other alloplastic materials that may lead to foreign body reactions. In addition, transplanted adipose tissue should not be considered only a means of filling, but also a restorative procedure for damaged or fibrotic tissue. Recent studies have demonstrated that adipose tissue contains a high prevalence of multipotent cells that are able to differentiate into different lineages and are then capable of at least partially restoring a damaged tissue by inducing angiogenesis. They also have anti-inflammatory and immune-modulating properties that may play a role $(14,24,28,38,41,43)$.

In the present study, we applied the procedure of AFTG in the perioral region of patients with SSc who complained of a reduced mouth opening as a consequence of their disease. We evaluated whether this microsurgical procedure may significantly improve their mouth opening and function. We also investigated whether this procedure may induce some changes in the microvascular architecture and dermal structure of the treated skin area.

\section{MATERIALS AND METHODS}

\section{Patients}

We studied 20 patients fulfilling the American College of Rheumatology (formerly, the American Rheumatism Association) criteria for SSc (37) and classified as having diffuse cutaneous $\mathrm{SSc}(\mathrm{dcSSc})$ on the basis of the criteria of LeRoy et al. (20).

All subjects were females with median age 36.5 years (range $40 \pm 13$ years) and had a median disease duration of 8 years (range $10 \pm 6$ years). All of the patients were enrolled because of the presence of a reduced capacity in opening their mouth (maximum interincisal distance $<55 \mathrm{~mm}$ ) that had been causing difficulties in eating/ drinking and personal or dentist tooth care. Presence of severe extracutaneous involvement, treatment with immunosuppressive therapies (including prednisone equivalent $>10 \mathrm{mg}$ ) in the 3 months before enrollment time, current pregnancy, and lactation represented the exclusion criteria.

The study was approved by the ethical committee of the "Ospedale G. Pini, Milan, Italy," where the study was carried out and performed according to the criteria of the Helsinki declaration. A written informed consent was obtained from all enrolled subjects to participate in the study. The patients did not pay for their inclusion or treatment in this study.

\section{Autologous Fat Grafting Procedure}

The trochanteric area or the periumbilical abdominal region represented the donor sites, given the easier access to a sufficient amount of adipose tissue. Following Coleman's technique (9), and after the administration of $150 \mathrm{ml}$ of local modified Klein solution [containing $100 \mathrm{ml}$ of saline, $20 \mathrm{ml}$ of mepivacaine $2 \%$ (A.I.C., Monico SPA, Venezia, Italy), $20 \mathrm{ml}$ of ropivacaine $7.5 \mathrm{mg} / \mathrm{ml}$ (B. Braun Melsungen AG, Melsungen, Germany), $1 \mathrm{ml}$ of epinephrine (S.A.L.F. S.p.A. Laboratorio Farmacologico, Bergamo, Italy), and $5 \mathrm{ml}$ of sodium bicarbonate solution $1 \mathrm{mEq} / \mathrm{ml}$ (S.A.L.F. S.p.A. Laboratorio Farmacologico)], a skin incision was performed in the selected area. Adipose tissue was harvested through the same incision after local infiltration of anesthetic solution. The cannula (Coleman Kit $15 \mathrm{~cm} \times 12 \mathrm{ga}$; Mentor ${ }^{\mathrm{TM}}$, Johnson \& Johnson Company, New Brunswick, NJ, USA) used for fat sampling was connected with a luer-lock syringe (Terumo-B.D.Troge, Nike S.r.l., Milan, Italy). The syringe plunger is pulled at the top; this creates a slight negative pressure inside the syringe, which allows the removal of adipose tissue. After suture (Ethicon; Johnson \& Johnson Company), the area was managed with an elastic-compressive dressing (BSN Medical, Hamburg, Germany), which was kept in place for 5 days to prevent hematoma formation. The adipose tissue was centrifuged (Hettich EBA 20, Seneco Science, Milan, Italy) at $700 \times g$ for $3 \mathrm{~min}$ in sterile conditions. Only the intermediate layer containing adipocytes and stromal vascular component was used for AFTG and then injected using a blunt cannula (Coleman Style II, $9 \mathrm{~cm} \times 17 \mathrm{ga}$; Mentor ${ }^{\mathrm{TM}}$, Johnson \& Johnson Company). Two milliliters was injected into each area using many radiating passages at the subcutaneous level of selected perioral regions. Six areas, three in the upper lip and three in the lower lip (one central and two lateral for each lip), plus one area for each opposite mouth corner region were identified for the multiple injections (up to a total amount of $16 \mathrm{ml}$ each patient).

\section{Clinical Outcome Evaluation}

All patients were closely followed in the 2 weeks after the AFTG to record any possible side effect. A precise clinical assessment of the mouth opening was performed at baseline time and 1 and 3 months after the surgical procedure. Mouth opening capacity was evaluated by measuring at baseline and in each follow-up time the maximum interincisal distance and the mouth perimeter. This latter figure was derived by the ellipse geometrical formula, that is, $2 \pi \sqrt{ } a^{2}+b^{2} / 2$, where " $a$ " is the distance in the maximally opened mouth between upper and 
lower lip, and " $b$ " is the distance between the opposite lip commissures.

Three months after the treatment, the patients were asked to fill in a simple questionnaire where their degree of satisfaction could be expressed by a semiquantitative score (unsatisfied, mildly/moderately satisfied, rather satisfied, and very satisfied).

\section{Skin Hardness Assessment}

Skin hardness was measured using a handheld digital durometer (Rex Gauge Type OO, Buffalo Grove, IL, USA). Durometer measurements were expressed in standard durometer units $(0-100)$ in a continuous scale. Three consecutive measurements were taken in six fixed perioral sites before and at 1 and 3 months after the AFTG, with the patient in a supine position at any time of evaluation and maintaining the facial muscles completely relaxed. The final measure used for the data analysis was derived by the sum of the means of the three measurements at each site.

\section{Labial Capillaroscopy}

Videocapillaroscopy (VC) of the lower lip was performed using a computerized system and related software (Videocap 200-DS Medigroup, Milan, Italy) that is used for the nailfold VC. The VC assessment was carried out with the patients in a sitting position, under the same conditions of light intensity and room temperature $\left(23^{\circ} \mathrm{C}\right)$ by the same observer (D.S.) in each patient. During examination, the mucosal surface of the lower lip was maintained perpendicular to the optical axis of the microscope. Eight VC digital images were taken in each patient along the vermilion border (four in the right part of the lip and four in the left one). Applying a 200× magnification, a surface of $1.25 \mathrm{~mm}^{2}$ was taken in each image. Two independent observers (D.S. and E.Z.) blindly assessed the VC digital images by counting the number of visible capillary loops in every VC field. The mean number of the capillary loops in the eight images from each patient was then calculated. The rounded mean of the two observer mean counts represented the final value of each VC assessment taken into account for the statistical analysis.

\section{Histological and Immunohistochemical Examination}

Skin biopsies were obtained from all of the patients in the lip commissure area at baseline and 3 months after the AFTG. The biopsies were fixed in 10\% neutral-buffered formalin, pH 7.2-7.4, (Diapath, Microstain Division, Martinengo-Bergamo, Italy) and routinely processed for paraffin embedding. Skin serial sections were obtained from formalin blocks, and 4- $\mu \mathrm{m}$ tissue sections were used for hematoxylin (Harris' hematoxylin nuclear staining; Bio-optica S.p.a., Milano, Italy) and eosin (Eosin G or Y alcoholic solution 0.5\%; Diapath, Microstain Division, Martinengo-Bergamo, Italy) (H\&E) and immunohistochemical $(\mathrm{IH})$ staining.

IH staining was performed by BenchMark ULTRA automated slide stainer (Ventana, Inc., Tucson, AZ, USA), and ultraView Universal DAB Detection Kit (Ventana, Inc.), and by using anti-cluster of differentiation 34 (CD34) and -anti-CD31 antibodies (provided concentration is optimized for use; clone Qbend/10 and clone JC70, respectively; Ventana, Inc.), as markers of endothelial cells. Three independent pathologists blindly performed a semiquantitative evaluation of both h/e and IH stained sections. Dermoepidermic junction (DEJ) flattening was scored on H\&E-stained sections as $0=$ absence of flattening, $1=$ mild flattening, $2=$ moderate flattening, and $3=$ severe flattening. Microvascular density (MVD) was assessed by counting the absolute number of $\mathrm{CD} 31^{+}$and $\mathrm{CD} 34^{+}$vessels per high-power field (HPF). Three areas per tissue section were selected on the basis of their higher density of positive elements. The average number of $\mathrm{CD} 31^{+}, \mathrm{CD} 34^{+}$ vessels per HPF were calculated and scored as follows: $1 \leq 5 / \mathrm{HPF} ; 2=5-10 / \mathrm{HPF}$, and $3 \geq 10 / \mathrm{HPF}$. For both MDV and DEJ evaluation, when the scores given by the observers were all different, the median value was the one taken into account for the statistical analysis. In the case that two out of the three observers gave the same score, this value was considered for statistics.

\section{Statistical Analysis}

Comparison between values at baseline time and those at 1 and 3 months after treatment was performed by paired $t$ test and Wilcoxon's test for continuous and noncontinuous variables, respectively. In view of the multiple comparisons made, only values of $p<0.01$ were regarded as statistically significant. Interrater agreement between the counts of capillaries in the VC stored images obtained by the two observers was performed by weighted $k$ statistics.

Data analysis was carried out using an SPSS statistical package (version 11.0.3, IBM, Chicago, IL, USA).

\section{RESULTS}

\section{Clinical Outcomes}

All patients treated presented a favorable outcome with improvement in subjective wellness of the skin in the perioral areas. When the patients were asked to express their overall personal opinion on the procedure they had undergone and on its effectiveness, $80 \%(16 / 20)$ and $20 \%$ (4/20) of them claimed to be very satisfied and rather satisfied, respectively. Figure 1 shows an example of aesthetic changes obtained after surgical treatment. The only complications noticed were small areas of ecchymosis, which rapidly disappeared within the following 2 weeks. No postsurgical local or systemic infections were recorded. 

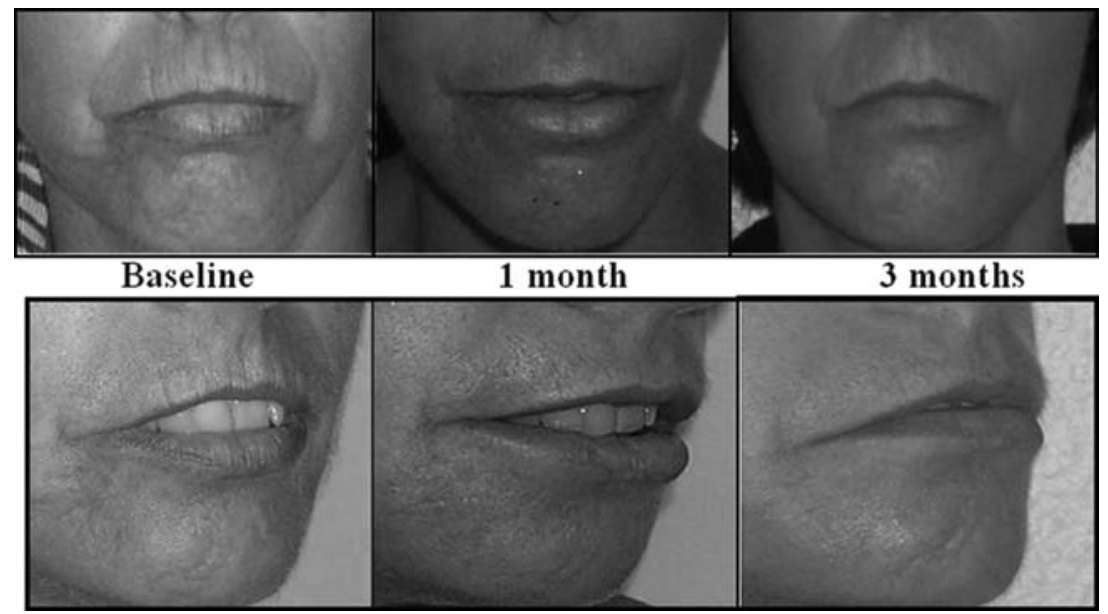

Figure 1. Aesthetic changes in the perioral region of a patient after autologous fat grafting (AFTG). One month and, more evidently, 3 months after treatment, the lip skin folds disappeared, and a complete closure of the mouth was possible, since a spontaneous contact between superior and inferior lip was allowed.

The maximum interincisal distance of the patients did not significantly change from baseline at 1-month assessment (mean variation -0.3 , ranging from -6.1 to 3.0 , $p=\mathrm{ns}$ ). On the contrary, a statistically significant increase of this measure was observed at 3 months (mean increase $2.63 \mathrm{~mm}$, ranging from 0.1 to $6 \mathrm{~mm}$ with respect to baseline value, paired $t$ value $7.83, p<0.001)$.

Similarly, after AFTG, we observed a gradual statistically significant increase in the mouth perimeter measurement from baseline to 1 month (mean increase $4.6 \mathrm{~mm}$, ranging from -0.6 to $11.8 \mathrm{~mm}$; paired $t$ test value 5.7 , $p<0.0001$ ) and to 3 months assessment (mean increase $9.2 \mathrm{~mm}$, ranging from -2.3 to $15.4 \mathrm{~mm}$; paired $t$ test value 8.6, $p<0.0001$ ) (Fig. 2).

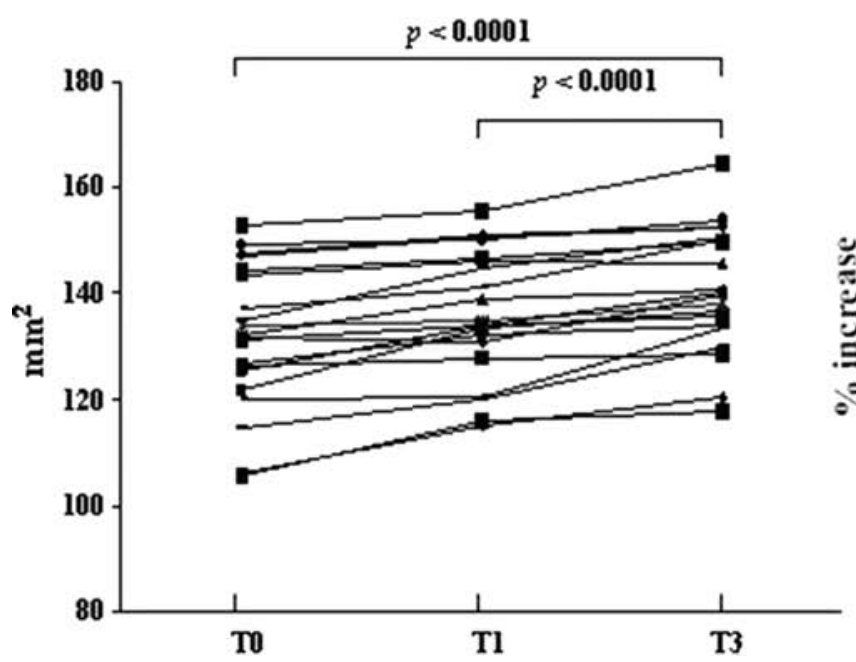

When we compared patients with more drastically reduced values of mouth perimeter at baseline time ( $\leq 126 \mathrm{~mm})$ to those with less compromised mouth opening (>126 mm) (respectively, 8 and 12 patients), the percentage of increase in perimeter values was significantly higher in the former group (12.4\% vs. 5.07\%; Student's $t$ test, $p<0.0001)$.

\section{Durometer Measurements in SSc Perioral Areas Before and After Lipostructure}

With regard to perioral skin hardness, durometer measurements showed that there was no significant change in durometer scores 1 month after AFTG. However, after 3 months, durometer scores were significantly decreased in

Figure 2. Perimeter measures at before and after AFTG. Perimeter measures at baseline and 1 and 3 months after AFTG expressed as absolute values (left side) and percent variations $( \pm 95 \% \mathrm{CI})$ from baseline (right side) are shown. A statistically significant difference was observed from baseline values to 1-month and 3-month values, and from 1-month and 3-month values $(p<0.0001$; paired $t$ values are detailed in the text). 
comparison to both the baseline and 1-month evaluation (paired $t$ test values 6.12 and 10.7, respectively, and $p<0.0001$ in both cases).

\section{Labial Capillaroscopy}

The microvascular architecture in SSc patients was characterized at baseline by a wide vascular disorganization with abnormalities of the capillary loops (Fig. 3a). Three months after AFTG, a significantly increased number of capillaries was observed (mean increase of 14.2 capillary loops, ranging from 4.25 to 25.0 , paired $t$ test value 8.0; $p<0.0001$ ), with an evidently more regular organization of microvascular architecture (Fig. 3b). The interrater agreement between the two observers who counted the number of capillaries in VC images was very good (weighted $k=0.905$ ).
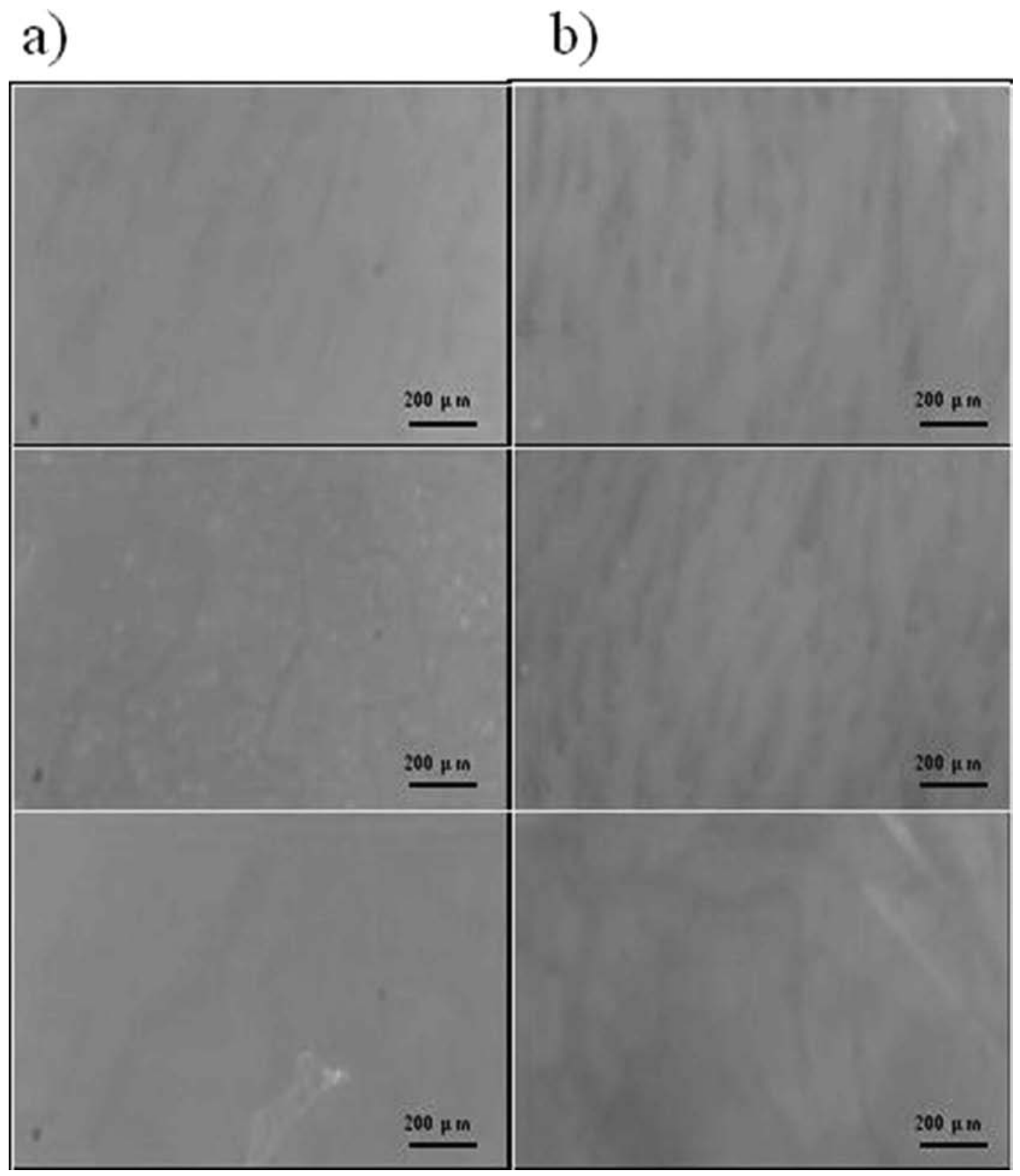

Figure 3. Changes in labial videocapillaroscopy (VC) patterns after AFTG. (a) At baseline, a wide vascular architectural disorganization with abnormalities of the capillary loops and reduced capillary number is observed. (b) After treatment, VC images show an increased number of capillaries with more regular organization of microcirculation. 


\section{Histopathological Findings}

Morphological evaluation of the biopsies on H\&E sections showed a reduction of DEJ flattening with epidermal crest elongation and a noticeable reconstruction of normal ridge pattern and dermal papillae in posttreatment samples when compared to baseline sections (Fig. 4).

The morphological changes were confirmed by the semiquantitative assessment. At baseline, the DEJ scores ranged from 2 to 3 and decreased to values ranging from 0 to 2 at the 3-month observation time (Wilcoxon's $Z$ value $4.02, p<0.0001)$.

The increase of MVD evaluated by scoring the $\mathrm{IH}$ changes in skin biopsy was highly significant $(Z=4.089$, $p<0.0001$ for $\mathrm{CD} 4^{+}$staining), with values ranging from 1 to 2 at baseline and from 3 to 4 at the 3 -month evaluation time. Identical figures were obtained considering the $\mathrm{CD} 31^{+}$-stained sections (Fig. 5).

\section{DISCUSSION}

In this study, AFTG has been performed in the perioral area of patients with SSc who complained of a reduction of their mouth opening and, consequently, difficulties in common daily living activities, like eating, drinking, and taking care of their teeth. We tried to apply this procedure taking into account previous positive therapeutic experiences on the treatment of other clinical conditions characterized by regional skin sclerosis or atrophy, like postirradiation dermatitis, faciocranial deformities, postburning or postsurgical sequelae $(6,8,19,32,34)$. The present study demonstrates that this microsurgical procedure is able to induce after 1 month but, more evidently after 3 months, a significant improvement of the mouth circumference and opening capacity. This increase is more evident in patients starting from a more strongly reduced mouth opening than in those where this measure

\section{Baseline}

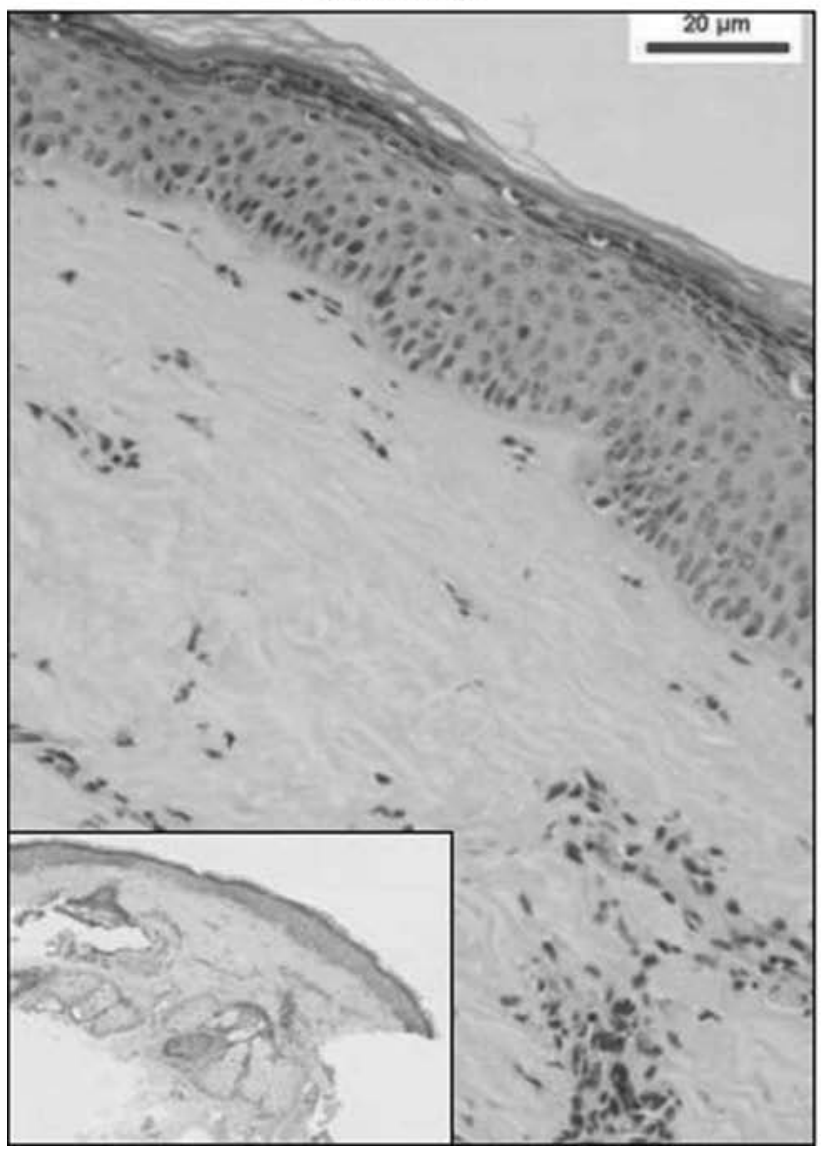

3 months

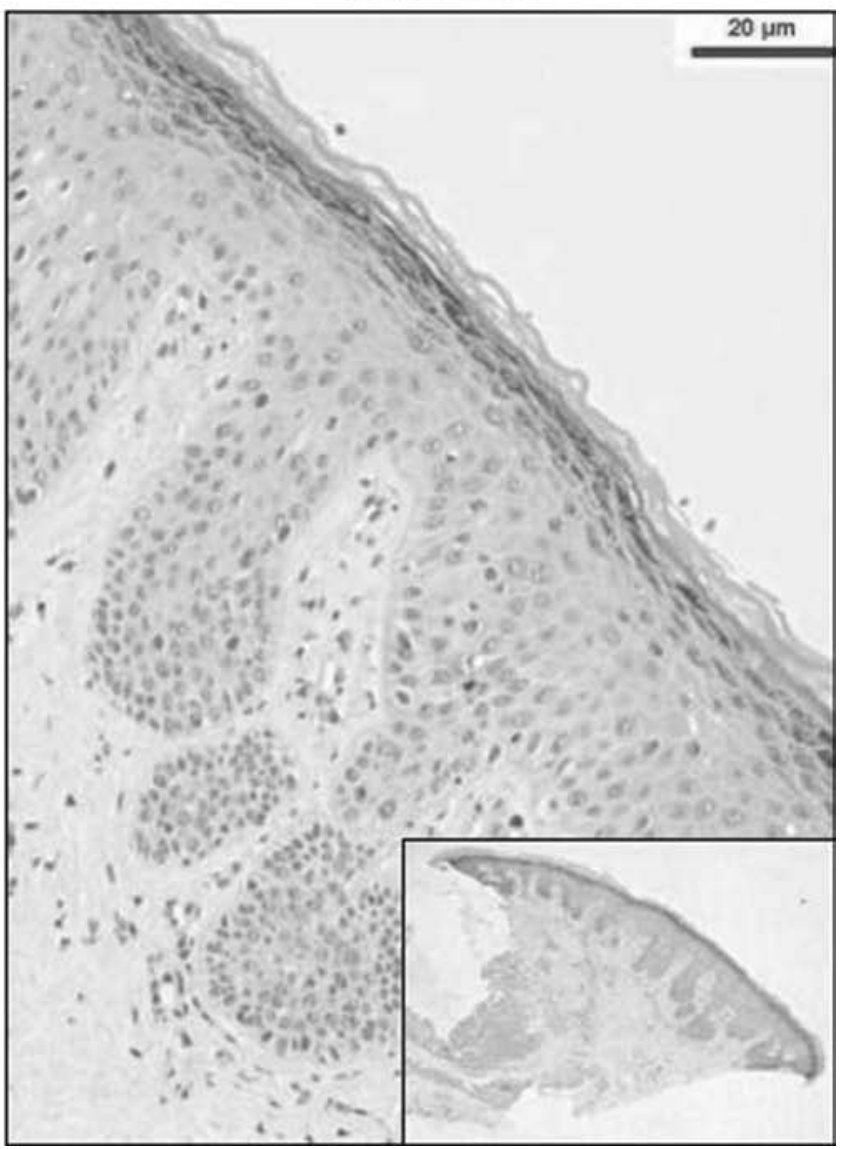

Figure 4. Example of ridges and dermal papillae in a pre- and posttreatment biopsy. Reconstruction of an example of ridges and dermal papillae in a posttreatment biopsy (right) compared to baseline (left) (hematoxylin and eosin staining; original magnification: $20 \times$, inset $4 \times$ ) (patient No. 3). 


\section{Baseline}
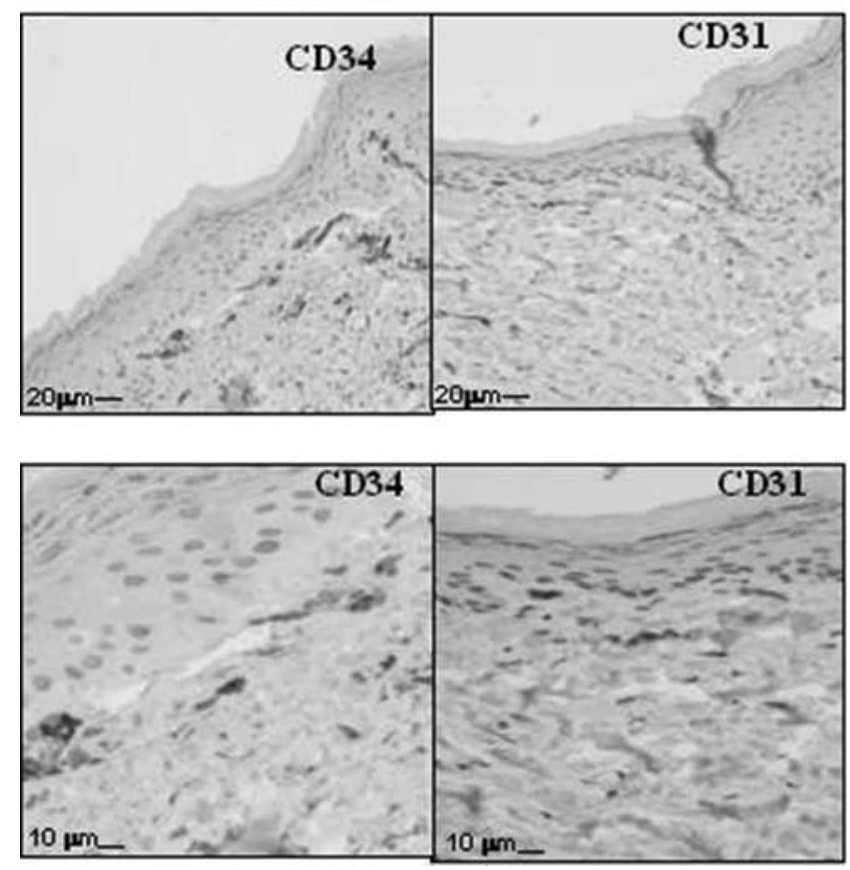

3 months
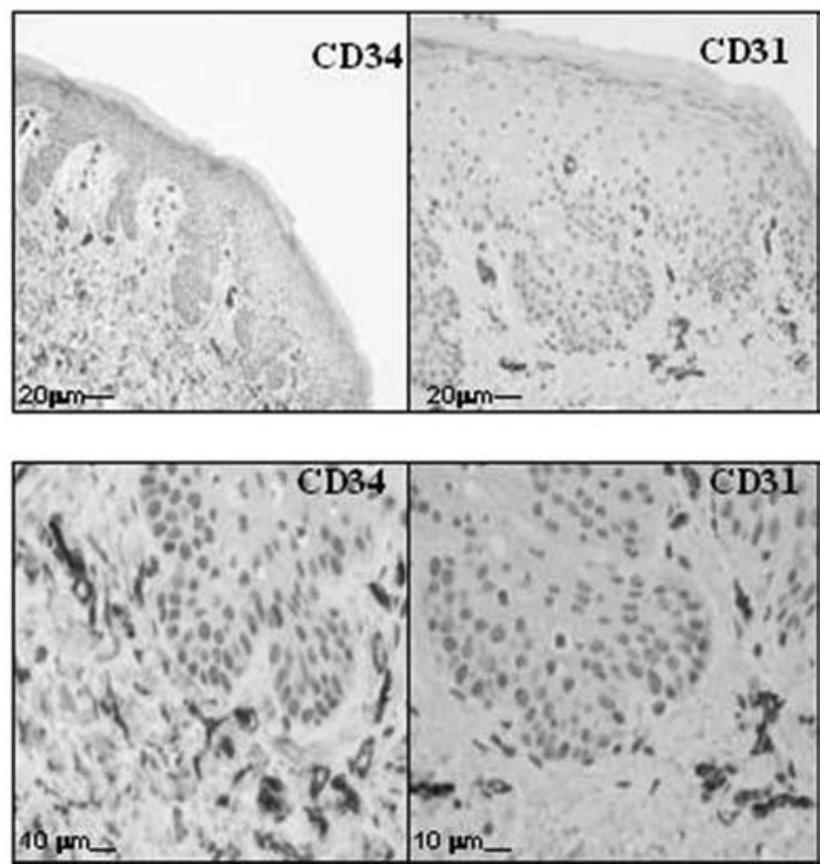

Figure 5. Increased CD34 and CD31 staining of small dermal vessels pre- and posttreatment. Increased number of cluster of differentiation 31 (CD31) and CD34 immunohistochemically stained small dermal vessels in a posttreatment sample (right side of the figure) in comparison to baseline (left side of the figure) (original magnification: $20 \times$ and 40x; patient No. 10).

was less quantitatively compromised at the enrollment time. Furthermore, the elasticity parameters of the perioral skin also appear to be significantly improved 3 months after the treatment, while after the first month they are unchanged or slightly worsened. Besides the improvement in the aesthetic and functional aspects we observed, the therapeutic procedure adopted in this study appears to be able to induce neoangiogenesis in the lip skin, as demonstrated by the evident changes of the VC and dermal immunohistochemical features after the treatment. Moreover, an at least partial restoration of the skin structure appears evident when the number and aspects of dermal papillae were examined in serial biopsies of the grafted region. Finally, it is worth noting that this minimally invasive surgical procedure was well accepted by the patients and did not induce any noticeable side effect.

Until now, treatment of scleroderma microstomia includes surgical bilateral commissurotomies (7) or a nonsurgical facial stretching program approach $(21,36)$. Besides the advantages obtained by these nonsurgical methods in terms of improvement of mouth opening capacity, no data are available regarding the beneficial effects that these procedures can induce in the cutaneous and subcutaneous SSc-related abnormalities. In addition, the observed functional improvement seems to be largely dependent on the continuity and repetition of exercises.
This is the first report in which this kind of therapeutic approach has been used to treat regional sclerotic changes in SSc. Some cases of linear scleroderma and morphea have been reported in which AFTG has been successfully used $(10,17,18,33)$. Other cases of localized scleroderma, as well as other patients with different atrophic or sclerotic skin abnormalities, have been alternatively treated by transplantation of ATDMSCs (35). Since similar results were reported by using AFTG or ATDMSC transplantation, one can argue that the two techniques could be considered interchangeable. In addition, taking into account the results of both previous and present reports, one could also conclude that the effectiveness of these procedures cannot be ascribed only to the filling effect, which is known to have a short duration $(25,26)$, but rather to the activation of various biological mechanisms (16), which could induce the morphological changes we observed. The fact that elasticity parameters slightly worsened after a month (a probable effect of the filling-related volumetric expansion), but strongly improved after 3 months, exactly in the same time when the most significant changes in mouth opening measures, the histological modifications, and microvascular modifications became more evident, seems to strongly support this assumption. In addition, even the observation that the result of grafting was not proportional to the baseline grade of 
reduction in mouth overture suggests that the functional improvement we obtained is not solely related to the volumetric filling effect. The long-term effects of this kind of treatment are at the moment unknown. However, some preliminary data at 6-month follow-up time, presently available in a small part of the enrolled patients, demonstrates that, although the filling effect of the procedure is almost completely lost, the improvement of mouth opening and function is substantially retained.

Different hypotheses can be raised to explain how adipose tissue grafting is effective in clinical conditions like localized and diffuse scleroderma. Recent studies demonstrated that, in fat tissue, a stem cell population is identifiable within the adipose stromal compartment. This cell population, defined as ATDMSCs, possesses many potentialities that are usually recognized to be a distinctive property of the bone marrow mesenchymal stromal cells (BMMSCs). ATDMSCs as well as BMMSCs are multipotent cells able to differentiate, under predefined conditions, into various cell types like adipocytes, chondrocytes, osteoblasts, myoblasts, and endothelial cells (2). Furthermore, it has been recently demonstrated that the mesenchymal stem cells (MSCs) of any origin can exert potent immunosuppressive, anti-inflammatory, and antifibrotic effects (39), and therefore their therapeutic use has been proposed. Preliminary investigations have been carried out in different autoimmune diseases like multiple sclerosis, Crohn's disease, systemic lupus, type 1 diabetes mellitus, systemic vasculitides, SSc, and in graft-versushost disease $(39,42)$. In addition, because of their potential angiogenic effects, MSCs have been proposed to be useful in the treatment of various vascular ischemic conditions (31).

The immunosuppressive effects of ATDMSCs have been mainly ascribed to their ability to inhibit, via multiple mechanisms, the proliferation of T- and B-cells. The anti-inflammatory effects of these cells are probably due to their capacity to downregulate the release of proinflammatory cytokines from $\mathrm{T}$ - and B-lymphocytes, natural killer (NK) cells, and dendritic cells. The anti-inflammatory effects may also be due to possible downregulation of different degrading enzymes like collagenase and gelatinase, which are overexpressed in inflammatory responses $(41,42)$. Moreover, the antifibrotic effects of adiponectin as a counterregulator of fibrotic gene expression and transforming growth factor- $\beta$ (TGF- $\beta$ ) signaling have been recently underlined (11). Finally, the fact that ATDMSCs are able to differentiate into endothelial cells and release angiogenetic factors supports their potential utility in promoting angiogenesis in surrounding tissues (38).

Progressive fibrotic changes, progressive vascular architecture derangement, and loss of skin and internal organs, like lung and heart, are undoubtedly the main and more critical pathological manifestation in patients with SSc and represent the most important therapeutic challenge in the management of this disease (4). However, treatment of pathological processes, which are active in generating sclerotic evolution and vascular desertification in SSc, remains an almost completely unsolved issue. Different therapeutic approaches have been sought by using immunosuppressive, anti-inflammatory, and chelating agents, but none of these treatments were clearly effective (29). Even more modern approaches using different biological agents able to interfere with key cytokines in the fibroblast lineage activation, like platelet-derived growth factor (PDGF) and transforming growth factor- $\beta 1$ (TGF- $\beta 1$ ), or new antifibrotic agents, like relaxin, or tyrosine kinase inhibitors did not show any really proven efficacy (3, 27,30).

On the contrary, there is clear evidence that autologous stem cell transplantation (ASCT) can be an effective therapeutic alternative in patients with dcSSc $(5,12,40)$. Besides the action of conditioning immunosuppressive therapy in ablating autoimmune response and strongly reducing fibroblast activation (1), it has been postulated that the ASCT effectiveness can be at least in part ascribed to the capacity of the repopulating stem cells to induce a neoangiogenesis in the sclerotic skin $(13,23)$.

The fact that, in the present study, the local grafting of fat tissue in the perioral area produced a neoproliferation of dermal capillaries and reduced the fibrotic changes with a partial restoration of the dermal structure may open new possibilities for the general therapeutic approach to this disease. The consequent debate on the possibility that the therapeutic effect of this procedure might be only ascribed to the on-site transplantation of the ATDMSCs or, alternatively, to the whole fat tissue grafting wherebesides the ATDMSCs - mature adipocytes and other biological mediators and growth factors are potentially active, remains, at the moment, purely speculative.

ACKNOWLEDGMENT: The authors declare no conflicts of interest.

\section{REFERENCES}

1. Abrahamsson, S.; Muraro, P. A. Immune re-education following autologous hematopoietic stem cell transplantation. Autoimmunity 41:577-584; 2008.

2. Barry, F. P.; Murphy, J. M.; Mesenchymal stem cells: Clinical applications and biological characterization. Int. J. Biochem. Cell Biol. 36:568-584; 2004.

3. Beyer, C.; Distler, O.; Distler, J. H. Innovative antifibrotic therapies in systemic sclerosis. Curr. Opin. Rheumatol. 24: 274-280; 2012.

4. Bhattacharyya, S.; Wei, J.; Varga, J. Understanding fibrosis in systemic sclerosis: Shifting paradigms, emerging opportunities. Nat. Rev. Rheumatol. 8:42-54; 2011.

5. Burt, R. K.; Shah, S. J.; Dill, K.; Grant, T.; Gheorghiade, M.; Schroeder, J.; Craig, R.; Hirano, I.; Marshall, K.; Ruderman, E.; Jovanovic, B.; Milanetti, F.; Jain, S.; Boyce, K.; Morgan, A.; Carr, J.; Barr, W. Autologous 
non-myeloablative haemopoietic stem-cell transplantation compared with pulse cyclophosphamide once per month for systemic sclerosis (ASSIST): An open-label, randomised phase 2 trial. Lancet 378:498-506; 2011.

6. Caviggioli, F.; Maione, L.; Vinci, V.; Klinger, M. The most current algorithms for the treatment and prevention of hypertrophic scars and keloids. Plast. Reconstr. Surg. 126: 1130-1131; 2010.

7. Chaffee, N. R. CREST syndrome: Clinical manifestations and dental management. J. Prosthod. 7:155-160; 1998.

8. Clauser, L.; Tieghi, R.; Consorti, G. Parry-Romberg syndrome: Volumetric regeneration by structural fat grafting technique. J. Craniomaxillofac. Surg. 38:605-609; 2010.

9. Coleman, S. R. Structural fat grafting: More than a permanent filler. Plast. Reconstr. Surg. 118(3 Suppl):108S-120S; 2006.

10. Conforti, G.; Tieghi, R.; Clauser, L. C. Frontal linear scleroderma: Long-term result in volumetric restoration of the fronto-orbital area by structural fat grafting. J. Craniofacial Surg. 23:263-264; 2012.

11. Fang, F.; Liu, L.; Yang, Y.; Tamaki, Z.; Wie, J.; Marangoni, R. G.; Bhattacharyya, S.; Summer, R. S.; Ye, B.; Varga, J. The adipokine adiponectin has potent antifibrotic effects mediated via adenosine monophosphateactivated protein kinase: Novel target for fibrosis therapy. Arthritis Res. Ther. 14(5):R229; 2012.

12. Farge, D.; Gluckman, E. Autologous HSCT in systemic sclerosis: A step forward. Lancet 378:460-462; 2011.

13. Fleming, J. N.; Nash, R. A.; McLeod, D. O.; Fiorentino, D. F.; Shulman, H. M.; Connolly, M. K.; Molitor, J. A.; Hensotrf, G.; Lafyatis, R.; Pritchard, D. K.; Adams, L. D.; Furst, D. E.; Schwartz, S. M. Capillary regeneration in scleroderma: Stem cell therapy reverses phenotype? PLoS One 3:e1452; 2008.

14. Gimble, J. M.; Katz, A. J.; Bunnel, B. A. Adipose-derived stem cells for regenerative medicine. Circ. Res. 100:1249-1260; 2007.

15. Hachulla, E.; Launay, D. Diagnosis and classification of systemic sclerosis. Clin. Rev. Allergy Immunol. 40:78-83; 2011.

16. Hsu, V. M.; Stransky, C. A.; Bucky, L. P.; Percec, I. Fat grafting's past, present, and future: Why adipose tissue is emerging as a critical link to the advancement of regenerative medicine. Aesthet. Surg. J. 32(7):892-899; 2012.

17. Karaaltin, M. V.; Akpinar, A. C.; Baghaki, S.; Akpinar, F. Treatment of "En coup de sabre" deformity with adiposederived regenerative cell-enriched fat graft. J. Craniofacial Surg. 23(2):e103-e105; 2012.

18. Lapierre, J. C.; Aasi, S.; Cook, B.; Montalvo, A. Successful correction of depressed scars of the forehead to trauma and morphea en coup de sabre by en bloc autologous dermal fat graft. Dermatol. Surg. 26:793-796; 2000.

19. Lee, S. K.; Kim, D. W.; Dhong, E. S.; Park, S. H.; Yoon, E. S. Facial soft tissue augmentation using autologous fat mixed with stromal vascular fraction. Arch. Plast. Surg. 39: 534-539; 2012

20. LeRoy, E. C.; Black, C.; Fleischmajer, R.; Jablonska, S.; Krieg, T.; Medsger, Jr., T. A.; Rowell, N.; Wollheim, F. Scleroderma (systemic sclerosis): Classification, subsets and pathogenesis. J. Rheumatol. 15:202-205; 1988.

21. Maddali-Bongi, S.; Landi, G.; Galluccio, F.; Del Rosso, A.; Miniati, I.; Conforti, M. L.; Casale, R.; Matucci-Cerinic, M. The rehabilitation of facial involvement in systemic sclerosis: Efficacy of the combination of connective tissue massage, Kabat's technique and kinesitherapy: A randomized controlled trial. Rheumatol. Int. 3(7):895-901; 2011.

22. Medsger, Jr., T. A. Natural history of systemic sclerosis and assessment of disease activity, severity, functional status, and psychologic well-being. Rheum. Dis. Clin. N. Am. 29: 255-273; 2003.

23. Miniati, I.; Guiducci, S.; Conforti, M. L.; Rogai, V.; Fiori, G.; Cinelli, M.; Saccardi, R.; Guidi, S.; Bosi, A.; Tyndall, A.; Matucci-Cerinic, M. Autologous stem cell transplantation improves microcirculation in systemic sclerosis. Ann. Rheum. Dis. 68:94-98; 2009.

24. Mizuno, H. Adipose-derived stem cells for tissue repair and regeneration: Ten years of research and a literature review. J. Nihon Med. Sch. 76:56-66; 2009.

25. Nguyen, A.; Pasyk, K. A.; Bouvier, T. N. Comparative study of survival of autologous adipose tissue taken and transplanted by different techniques. Plast. Reconstr. Surg. 85:378-386; 1990 .

26. Niechajev, I.; Sevchuk, O. Long-term results of fat transplantation: Clinical and histologic studies. Plast. Reconstr. Surg. 94:496-506; 1994.

27. Ong, V. H.; Denton, C. P. Innovative therapies for systemic sclerosis. Curr. Opin. Rheumatol. 22:264-272; 2010.

28. Poloni, A.; Maurizi, G.; Leoni, P.; Serrani, F.; Mancini, S.; Frontini, A.; Zingaretti, M. C.; Siquini, W.; Sarzani, R.; Cinti, S. Human dedifferentiated adipocytes show similar properties to bone marrow-derived mesenchymal stem cells. Stem Cells 30:965-974; 2012.

29. Quillinan, N. P.; Denton, C. P. Disease-modifying treatment in systemic sclerosis: Current status. Curr. Opin. Rheumatol. 21:636-641; 2009.

30. Ramos-Casals, M.; Fonollosa-Pla, V.; Brito-Zerón, P.; Sisó-Almirall, A. Targeted therapy for systemic sclerosis: How close are we? Nat. Rev. Rheumatol. 6:269-278; 2010.

31. Ren, G.; Chen, X.; Dong, F.; Li, W.; Ren, X.; Zhang, Y.; Shi, Y. Concise review: Mesenchymal stem cells and translational medicine: Emerging issues. Stem Cells Transl. Med. 1:51-58; 2012.

32. Rigotti, G.; Marchi, A.; Galié, M.; Baroni, G.; Benati, D.; Krampera, M.; Pasini, A.; Sbarbati, A. Clinical treatment of radiotherapy tissue damages by lipoaspirates transplant: A healing process mediated by adipose derived stem cells. Plast. Reconstr. Surg. 119:1409-1422; 2007.

33. Roh, M. R.; Jung, J. Y.; Chung, K. Y. Autologous fat transplantation for depressed linear scleroderma-induced facial atrophic scars. Dermatol. Surg. 34:1659-1665; 2008.

34. Salgarello, M.; Visconti, G.; Barone-Adesi, L. Fat grafting and breast reconstruction with implant: Another option for irradiated breast cancer patients. Plast. Reconstr. Surg. 129: 317-329; 2012.

35. Scuderi, N.; Ceccarelli, S.; Onesti, M. G.; Fieramonti, P.; Guidi, C.; Romano, F.; Frati, L.; Angeloni, A.; Marchese, C. Human adipose-derived stem cells for cell-based therapies in the treatment of systemic sclerosis. Cell Transplant. 22(5):779-795; 2013.

36. Shouffer, A. A.; Ninaber, M. K.; Beaart-Van De Voorde, L. J. J.; Van Der Giesen, F. J.; De Jong, Z.; Stolk, J.; Voskuyl, A. E.; Scherptong, R. W. C.; Van Laar, J. M.; Schuerwegh, A. J. M.; Huizinga, T. W. J.; Vliet Vlieland, T. P. M. Randomized comparison of a multidisciplinary team care program with usual care in patients with systemic sclerosis. Arthritis Care Res. 63(6):909-917; 2011.

37. Subcommittee for Scleroderma Criteria of the American Rheumatism Association Diagnostic and Therapeutic Criteria 
Committee. Preliminary criteria for the classification of systemic sclerosis (scleroderma). Arthritis Rheum. 23:581-590; 1980.

38. Takahashi, M. Adipose tissue: An alternative source for therapeutic angiogenesis. Circ. J. 76:1597-1598; 2012.

39. Tyndall, A.; Uccelli, A. Multipotent mesenchymal stromal cells for autoimmune diseases: Teaching new dogs old tricks. Bone Marrow Transplant. 43:821-828; 2009.

40. van Laar, J. M.; Farge, D.; Sont, J. K.; Naraghi, K.; Marjanovic, Z; Schuerwegh, A. J. The ASTIS trial: Autologous stem cell transplantation versus IV pulse cyclophosphamide in poor prognosis systemic sclerosis, first results. Ann. Rheum. Dis. 71(Suppl 3):15; 2012.
41. Yañez, R.; Lamina, M. L.; García-Castro, J.; Colmenero, I.; Ramírez, M.; Bueren, J. A. Adipose tissue-derived mesenchymal stem cells have in vivo immunosuppressive properties applicable for the control of the graft-versus-host disease. Stem Cells 12:3375-3382; 2006.

42. Yi, T.; Song, S. U. Immunomodulatory properties of mesenchymal stem cells and their therapeutic applications. Arch. Pharm. Res. 35:213-221; 2012.

43. Zuk, P. A.; Zhu, M.; Mizuno, H.; Huang, J.; Futrell, J. W.; Katz, A. J.; Benhaim, P.; Lorenz, H. P.; Hedrick, M. H. Multilineage cells from human adipose tissue: Implication for cell-based therapies. Tissue Eng. 7:211-228; 2001. 